\title{
Dexmedetomidine infusions and phenobarbital in the treatment of an unusual presentation of benzodiazepine-resistant alcohol withdrawal
}

\author{
Neil Shah ${ }^{*}$, Theresa Yang², Janine Vintch²
}

\begin{abstract}
${ }^{*}$ Correspondence
Neil Shah

1000 West Carson St Hospital Building:

1 East, Box 10 Torrance, CA 90509

Phone: 818-414-7765 Fax: 780-328-7713

Email: nshah@dhs.lacounty.gov

${ }^{1}$ Los Angeles County Harbor-UCLA Medical Center, Anesthesiology. 2 Harbor-UCLA Medical Center, Division of Respiratory and Critical Care Physiology and Medicine, Department of Medicine.

Received: Jun 13, 2019

Accepted: Aug 1, 2019

Published: Aug 8, 2019

(c) Neil Shah et al, 2019; licensee OA Journal of Case Reports. This is an Open Access article distributed under the terms of the Creative Commons Attribution License (http:// creativecommons.org/licenses/by/4.0), which permits unrestricted use, distribution, and reproduction in any medium, provided the original work is properly credited.
\end{abstract}

\begin{abstract}
Background: Alcohol withdrawal is a life-threatening condition characterized by a myriad of physiologic changes including tachycardia, hypertension, lowered seizure threshold, hallucinations, and potential for delirium tremens. Benzodiazepines remain the gold standard for treatment of alcohol withdrawal, although few studies have compared barbiturates to benzodiazepines as first-line treatment.

Methods: This study is a single patient chart review.

Results: Over the course of his hospital stay, in addition to receiving a continuous infusion of dexmedetomidine, the patient received a total of $389 \mathrm{mg}$ lorazepam, $650 \mathrm{mg}$ phenobarbital, $40 \mathrm{mg}$ haloperidol, $25 \mathrm{mg}$ quetiapine, $5 \mathrm{mg}$ midazolam, and $75 \mathrm{mg}$ diphenhydramine.

Conclusion: Phenobarbital is an effective first line agent for management of alcohol withdrawal and may be a safer and more effective treatment with lower rates of intubation and shorter hospital stays than benzodiazepines. It is particularly successful in patients who require high doses of benzodiazepines or ICU admission. Furthermore, the role of dexmedetomidine infusions in alcohol withdrawal remains unclear but may play a critical role in mitigating tachycardia and hypertension though it poses a risk of bradycardia and hypotension.
\end{abstract}

Keywords: Alcohol withdrawal, Dexmedetomidine, Precedex, Phenobarbital, Ativan, Lorazepam, CIWA, GABA channel.

\section{Introduction}

Alcohol withdrawal is life-threatening condition characterized with a myriad of physiologic changes including tachycardia, hypertension, lowered seizure threshold, hallucinations, and potential for delirium tremens. The pathophysiology of alcohol withdrawal is not entirely known but believed to involve attenuation of the GABAergic pathway and heightening of the glutaminergic pathway. The use of
GABA agonists, including benzodiazepines, has been the standard of care for treatment of alcohol withdrawal, although newer research has attempted to elucidate how inhibiting the glutaminergic response might help control withdrawal symptoms as well [1].

This individual is a 43-year-old, $63 \mathrm{~kg}$ male, with chronic alcohol use disorder who presented to an Emergency Department (ED) with right hip pain after 
falling from a ladder while intoxicated. He had no focal neurological findings, a negative skeletal survey, and no abnormalities were noted on full-body CT imaging. Initial laboratory evaluation revealed leukocytosis with neutrophilic predominance, lactic acidosis, elevated transaminases, acute kidney injury, and rhabdomyolysis. Of note, the total bilirubin, PT, and PTT were not elevated. He also had an elevated ethanol level $(26$, normal $<5$ ) but no recreational substances were detected.

\section{Materials and Methods}

All data was collected on a single patient through a retrospective chart review including vital signs, laboratory data and radiographic data, medication administration data, and documentation.

In the ED, his CIWA-AR score was retrospectively calculated to be 40 but was likely greater due to lack of documentation of all of the criteria. He received lorazepam, phenobarbital, and fentanyl. His heart rate subsequently decreased from 150 s to $120 \mathrm{~s}$, his blood pressure decreased from 191/149 to 123/81, and he became less agitated and less tremulous. He was admitted to the intensive care unit for management of severe alcohol withdrawal.

His ICU stay was complicated by agitation, delirium, tachycardia, and hypertension despite receiving high doses of lorazepam.

Prior to starting dexmedetomidine on day 4, lorazepam was increased from 4 to $6 \mathrm{mg}$ as needed with $116 \mathrm{mg}$ of lorazepam administered over the preceding 48 hours, hard restraints were applied, and a hospital emergency behavioral code was activated as our patient attempted to rip off his soft restraints, pulled and chewed on his intravenous lines, spat on and attempted to bite and kick staff members. Further, his heart rate raised from 80s-100s to 130s-140s. Due to these factors, a dexmedetomidine infusion was started. On day 6, he was calm, cooperative, and vocalized the importance of receiving medical care in the hospital. His average heart rate and mean arterial pressure decreased from $100 \mathrm{bpm}$ and $102 \mathrm{~mm} \mathrm{Hg}$ in the 24 hours prior to dexmedetomidine administration to $74 \mathrm{bpm}$ and $75 \mathrm{~mm} \mathrm{Hg}$ while receiving the infusion. In light of his clinical improvement, the dexmedetomidine infusion was stopped on day 6. He was discharged home on day 12 . The cumulative dose of all sedating medications the patient received during his admission is listed in Table 1.

Table 1: Cumulative doses of all sedating medications given to the patient.

\begin{tabular}{|l|l|}
\hline Medication & Dose \\
\hline$\alpha-2$ agonist & \\
\hline Dexmedetomidine & $\begin{array}{l}1.4 \mathrm{mcg} / \mathrm{kg} / \mathrm{hr} \text { administered for } 57 \\
\text { hours as a continuous infusion }\end{array}$ \\
\hline GABA agonist & $389 \mathrm{mg}$ \\
\hline Lorazepam & $5 \mathrm{mg}$ \\
\hline Midazolam & $650 \mathrm{mg}$ \\
\hline Phenobarbital & $40 \mathrm{mg}$ \\
\hline Dopamine antagonists & $25 \mathrm{mg}$ \\
\hline Haloperidol & \\
\hline Quetiapine & $75 \mathrm{mg}$ \\
\hline First generation H1 antagonist \\
\hline Benadryl
\end{tabular}

\section{Results and Discussion}

This patient's failure to adequately respond to a benzodiazepine monotherapy highlights the challenge in managing benzodiazepine-resistant alcohol withdrawal. Although few studies have documented the average benzodiazepine dose during alcohol withdrawal hospitalizations, an observational study by Martinez et al. found that among patients admitted for alcohol withdrawal, the average diazepam-equivalents among patients who were not pretreated with high-dose baclofen was 310 mg (vs. $398 \mathrm{mg}$ that our patient was given when converted to diazepam equivalents) [2]. Consequently, our patient's care necessitated the use of additional agents, including phenobarbital and a dexmedetomidine infusion.

When barbiturates are combined with benzodiazepines, there are additive effects as both act on the GABA receptors [3]. While benzodiazepines increase the frequency of GABA-mediated chloride channel opening, barbiturates increase the length of time that the channel stays open. A metanalysis by Yooshun et al. found that dual therapy with benzodiazepines and barbiturates allowed for better control of alcohol withdrawal symptoms, particularly in severe forms, and for a lower dose of each 
medication. Patients who received barbiturates did not have an increase in respiratory depression and in fact had lower rates of mechanical ventilation [4]. A study by Hack et al. of 19 patients who were admitted for alcohol withdrawal and required at least $50 \mathrm{mg}$ diazepam per hour found that 14 patients (73.7\%) failed benzodiazepine monotherapy and required barbiturates [5]. Our patient required 100 mg diazepam equivalents per hour and thus earlier introduction to phenobarbital may have been appropriate. Larger studies are needed to evaluate the role of combination therapy with benzodiazepines and barbiturates.

Barbiturate monotherapy is useful for treating alcohol withdrawal refractory to benzodiazepines. A cohort study by Tidwell et al. found that phenobarbital monotherapy was associated with a lower incidence of intubation compared to benzodiazepines under the CIWA-Ar protocol (1 [2\%] vs. 14 [23\%], $p<0.001$ ), a shorter ICU (2.4 vs. 4.4 days, $p<0.001$ ), and shorter hospital stay ( 4.3 vs. 6.9 days, $p=0.004$ ) [6]. More research is needed to identify predictors of patients who are more likely to fail benzodiazepine monotherapy and may benefit from receiving barbiturate monotherapy early in the hospital course.

Benzodiazepine and alcohol withdrawal are both known to independently cause delirium. Further, benzodiazepine use has been associated with paradoxical agitation. The rate of delirium and paradoxical agitation secondary to benzodiazepines has not been consistently reported [7]. A retrospective study by Moore et al. examined 85 patients admitted for alcohol withdrawal who were given benzodiazepines and subsequently developed delirium [8]. Each patient was then given flumazenil, a GABAergic antagonist, to reverse the effects of the benzodiazepines. 62 patients $(72.9 \%)$ experienced cognitive improvement after receiving flumazenil, while 2 (2.4\%) had worsening anxiety. These results suggest that delirium seen in alcohol withdrawal treated with benzodiazepines may often be secondary to benzodiazepine use.

The role of dexmedetomidine infusions in augmenting benzodiazepines in alcohol withdrawal has only recently been explored in the literature. Dexmedetomidine is an 2 agonist and thus does not potentiate the GABAergic effects, including respiratory depression, seen with benzodiazepines and barbiturates. However, animal studies have suggested a synergistic pharmacodynamic interaction between dexmedetomidine and benzodiazepines in terms of their anxiolytic effect, with variable interactions of cardiovascular and ventilator side effects. Dexmedetomidine is believed to treat alcohol withdrawal through damping the heightened adrenergic response that cause tremors, high blood pressure, tachycardia, and anxiety. Given that alcohol withdrawal predictably improves five to seven days after cessation of the last drink which was the same time that the patient was started on a dexmedetomidine infusion, it is unclear how much the drip may have been responsible for our patient's clinical improvement. A retrospective study by Beg et al. studied 77 patients admitted to the ICU for alcohol withdrawal and found that patients who received dexmedetomidine had better CIWA scores and required less benzodiazepine use in the first 24 hours after administration of dexmedetomidine [9]. However, there was no difference in the cumulative benzodiazepine dose given over the hospitalization. Further, patients who received dexmedetomidine had longer hospitalizations ( 8.9 days vs. 4.7, $p<0.01$ ) and ICU stays (2.9 days vs $1.4, \quad p<0.01)$. Four patients (10.5\%) were unable to tolerate dexmedetomidine due to bradycardia or hypotension. A prospective study by Mueller et al. examined 24 patients in alcohol withdrawal with a CIWA score of $>15$ despite receiving $16 \mathrm{mg}$ of lorazepam over 4 hours [10]. This study also found less benzodiazepine usage while receiving dexmedetomidine and no overall difference in benzodiazepines usage. Further, there was no difference in hospital lengths of stay. Similarly, our patient's agitation, delirium, and tremors subjectively improved after receiving dexmedetomidine. While receiving dexmedetomidine compared to the prior 24 hours, he required less lorazepam (1.4 vs. $3.2 \mathrm{mg}$ per hr), had a lower heart rate (73.6 bpm vs. 100.1 in the 24 hours prior), and had lower mean arterial pressure (74.9 mm Hg vs. 102 in the 24 hours prior) [11].

\section{References}

1. Rao PS, Bell RL, Engleman EA, et al. (2015) Targeting glutamate uptake to treat alcohol use disorders. Front Neurosci. 9:144.

2. Martinez L, Vorspan F, Xavier D, et al. (2017) An observational study of benzodiazepine prescription during inpatient alcohol detoxification for patients with versus without chronic 
pretreatment with high dosage baclofen. Fundamental \& Clinical Pharmacology. 32:200-205.

3. Ashton H. (1995) Toxicity and adverse consequences of benzodiazepine use. Psychiatric Annals.25:158-165.

4. Mo Y, Thomas MC, Karras GE Jr. (2016) Barbiturates for the treatment of alcohol withdrawal syndrome: A systematic review of clinical trials. J Crit Care. 32:101-107.

5. Hack JB, Hoffmann RS, Nelson LS. (2006) Resistant alcohol withdrawal: does an unexpectedly large sedative requirement identify these patients early? J Med Toxicol. 2:55-60.

6. Tidwell WP, Thomas TL, Pouliot JD. (2018) Treatment of Alcohol Withdrawal Syndrome: Phenobarbital vs CIWA-Ar Protocol. Am J Crit Care. 27:454-460.

7. Mancuso CE, Tanzi MG, Gabay M. (2004) Paradoxical reactions to benzodiazepines: literature review and treatment options. Pharmacotherapy. 24:1177-1185.
8. Moore PW, Donovan JW, Burkhart KK, et al. (2014) Safety and efficacy of flumazenil for reversal of iatrogenic benzodiazepine-associated delirium toxicity during treatment of alcohol withdrawal, a retrospective review at one center. J Med Toxicol.10:126-132.

9. Beg M, Fisher S, Siu D, et al. (2016) Treatment of Alcohol Withdrawal Syndrome with and without Dexmedetomidine. Perm J. 20:49-53.

10. Mueller SW, Preslaski CR, Kiser TH. (2014) A randomized, double-blind, placebo-controlled dose range study of dexmedetomidine as adjunctive therapy for alcohol withdrawal. Crit Care Med. 42:1131-1139.

11. Bol CJ, Vogelaar JP, Tang JP, et al. (2000) Quantification of pharmacodynamic interactions between dexmedetomidine and midazolam in the rat. J Pharmacol Exp Ther. 294:347-355. 